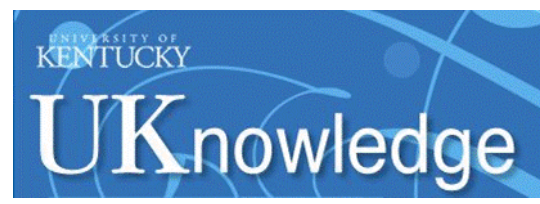

University of Kentucky

UKnowledge

10-2019

\title{
Multiple Sclerosis Outcomes after Cancer Immunotherapy
}

\author{
Catherine R. Garcia
}

University of Kentucky

Rani Jayswal

University of Kentucky, rani.jayswal@uky.edu

Val R. Adams

University of Kentucky, val.adams@uky.edu

Lowell B. Anthony

University of Kentucky, lowell.anthony@uky.edu

John L. Villano

University of Kentucky, jlvillano@uky.edu

Follow this and additional works at: https://uknowledge.uky.edu/markey_facpub

Part of the Internal Medicine Commons, Neurology Commons, Neurosurgery Commons, Oncology Commons, and the Pharmacy and Pharmaceutical Sciences Commons

Right click to open a feedback form in a new tab to let us know how this document benefits you.

\section{Repository Citation}

Garcia, Catherine R.; Jayswal, Rani; Adams, Val R.; Anthony, Lowell B.; and Villano, John L., "Multiple Sclerosis Outcomes after Cancer Immunotherapy" (2019). Markey Cancer Center Faculty Publications. 158.

https://uknowledge.uky.edu/markey_facpub/158

This Review is brought to you for free and open access by the Markey Cancer Center at UKnowledge. It has been accepted for inclusion in Markey Cancer Center Faculty Publications by an authorized administrator of UKnowledge. For more information, please contact UKnowledge@lsv.uky.edu. 


\section{Multiple Sclerosis Outcomes after Cancer Immunotherapy}

Digital Object Identifier (DOI)

https://doi.org/10.1007/s12094-019-02060-8

\section{Notes/Citation Information}

Published in Clinical and Translational Oncology, v. 21, issue 10.

(c) 2019 Springer Nature Switzerland AG.

This is a post-peer-review, pre-copyedit version of an article published in Clinical and Translational Oncology. The final authenticated version is available online at: https://doi.org/10.1007/

s12094-019-02060-8 
Catherine R. Garcia: catherinegarcia@uky.edu; Rani Jayswal: rani.jayswal@uky.edu; Val Adams: val.adams@uky.edu; Lowell B Anthony: lowell.anthony@uky.edu; John L. Villano: jlvillano@uky.edu

\section{Abstract}

Introduction: Neurological immune-related adverse events are a rare but potentially deadly complication after immune checkpoint inhibitor (ICI) treatment. As multiple sclerosis (MS) is an immune mediated disease it is unknown how ICI treatment may affect outcomes.

Methods: We analyzed the United States Food and Drug Administration (FDA) Adverse Event Reporting System (FAERS) database for pembrolizumab, atezolizumab, nivolumab, ipilimumab, avelumab, and durvalumab two years prior their FDA approval until December 31, 2017, to include all cases with confirmed diagnosis/relapse of MS. We also included cases reported in the literature and a patient from our institution.

Results: We identified 14 cases of MS with median age of presentation of 52 years. Indications for ICI included melanoma in 7 (36.36\%) cases, non-small cell lung carcinoma in $2(18.18 \%)$ cases, 1 case $(9.09 \%)$ each of pleural mesothelioma, renal cell carcinoma, and colorectal cancer, and unreported in $2(18.18 \%)$ cases. History of MS was confirmed in $8(57.1 \%)$ cases. Median time to beginning of symptoms was 29 days with rapid disease progression; 2 patients died due to their relapse. Median time for symptom resolution was 8 weeks. Outcomes did not vary by comparing CTLA-4 and PD-1/PD-L1 inhibitors.

Corresponding Author: John L. Villano, MD, PhD, University of Kentucky, Markey Cancer Center, 800 Rose Street, CC446, Lexington, KY 40536-0293, Tel: 859-323-0405, Fax: 859-257-7715.

AUTHOR CONTRIBUTIONS

Catherine Garcia: Methodology, conceptualization, investigation, data curation, writing — original draft, and writing — review and editing. Rani Jayswal: Formal analysis, writing—review and editing. Val Adams: Conceptualization, writing— review and editing. Lowell Anthony: Conceptualization, writing — review and editing. John Villano: Methodology, conceptualization, writing — review and editing.

Publisher's Disclaimer: This Author Accepted Manuscript is a PDF file of an unedited peer-reviewed manuscript that has been accepted for publication but has not been copyedited or corrected. The official version of record that is published in the journal is kept up to date and so may therefore differ from this version.

Supplementary Data: None

Disclosures: No disclosures.

Conflicts of interest: The authors have no conflicts of interest.

Ethical statement: Patient consent was not obtained from our institutional case since the patient passed away many years prior to preparation of this manuscript. Data is de-identified in FAERS and patient consent is not applicable. Our study does not include any studies with animals performed by any of the authors. 
Conclusions: Reported MS relapses after ICI are rare but the adverse events described include rapid neurologic progression and death. Larger and prospective studies are warranted to assess disability and long-term outcomes and outweigh the risks of starting immunotherapy in patients with MS.

\section{Keywords}

1-Multiple sclerosis; 2-immune checkpoint inhibitors; 3-immune related adverse events

\section{INTRODUCTION}

Immune checkpoint inhibitors (ICI) include CTLA-4 and PD-1/PD-L1 inhibitors and have recently become the standard of care for a growing list of cancers, including melanoma, nonsmall cell lung cancer, renal cell carcinoma, Hodgkin lymphoma, head and neck cancer, urothelial carcinoma, and microsatellite instability-high solid tumors $[2,4,5,9,16,24,28$, $34,35]$. Immunotherapies differ from conventional chemotherapy as they are not directly cytotoxic, but are designed to engage the immune system to generate antitumor activity[13]. CTLA-4 and PD-1/PD-L1 are ligands to the B7 costimulatory pathway that mediate T-cell inhibition[19]. Due to their mechanisms of action, ICI have been associated with a unique spectrum of immune related adverse events (irAEs) by deregulating the response of T cells to antigens presented by normal cells. A possibility exists for immune mediated diseases, such as multiple sclerosis (MS), to be triggered or significantly aggravated with the use of these medications[6, 10]. However, knowledge on the neurological irAEs is still insufficient due to the novelty of these drugs.

MS affects more than 400,000 people in the United States, presenting with an array of clinical presentations that include vision loss, limb weakness, sensory abnormalities, or ataxia[1]. Several studies support an inflammatory patient-dependent immune-mediated component for MS[27, 43], with genetic and environmental risk factors identified[3], and a T-cell mediated pathogenesis mechanistically similar to ICI[22, 39].

Neurological complaints are one of the most common reasons for hospital admission in cancer patients[31]. Severe neurological irAEs with ICI therapy have been reported in less than $1 \%$ of patients[23, 40,42,45]. The majority of neurological irAEs manifest predominantly as peripheral neuropathy and are generally associated with a benign course $[8$, 25]. MS is the most common cause of permanent disability in young adults[30, 33], with limited response to treatment. Future functional impairment is a major concern if a relapse develops during ICI therapy, more so than with other neurological irAEs.

No study has focused on the outcomes of MS patients after ICI therapy, and with an increasing approval of ICI use safety in these patients will be an important clinical question. Due to the heterogeneity of MS, response after ICI may be unpredictable. We analyzed the outcomes of documented cases of MS relapse after treatment with ICI in a comprehensive analysis of the available published literature, the Food and Drug Administration (FDA) Adverse Event Reporting System (FAERS) database, and our institutional experience. 


\section{METHODS}

We present one case from our institution, a review of the published literature, and the FAERS database for patients who developed MS relapse during cancer therapy with ICI.

FAERS data was provided by the FDA upon request.

\section{Search strategy}

Institutional-We performed a retrospective analysis of cancer patients that received at least one cycle of pembrolizumab, atezolizumab, nivolumab, ipilimumab, avelumab, and/or durvalumab during any part of their cancer treatment and that had a history of MS that developed symptoms during or after their therapy with ICI. Only 1 patient was identified from this search.

FAERS-We analyzed the FAERS database for pembrolizumab, atezolizumab, nivolumab, ipilimumab, avelumab, and durvalumab from 2 years before their FDA approval until December 31, 2017, to include all cases with confirmed diagnosis of MS. After case identification, detailed reports were requested to the FDA and information is presented as available in those reports. Multiple reports that described the same patient were grouped together.

FAERS is an international database maintained by the FDA that collects de-identified information on adverse event reports, medication error, and product quality complaints. Reports are limited to the information disclosed by the reporter, which includes health care providers, drug manufacturers, and consumers. Information included in these reports details drug exposure, disease indications, and clinical outcomes[36, 37]. FAERS is a reliable source of information that has been used extensively by researchers and our group in several areas to describe post-marketing surveillance of medications[15]. It provides a unique opportunity to assess cases globally with detailed reports of their event.

Based on FDA regulations, outcomes are reported in the following categories: congenital anomaly/birth defect, death, disability, hospitalization, life threatening, other serious important medical event, and required intervention to prevent permanent impairment/ damage. OT is defined as an outcome that does not fit other categories, but may jeopardize patients requiring medical or surgical intervention to prevent one of the other outcomes.

Literature-We performed a systematic review of the literature using the PubMed database. We searched for all published literature regarding MS and nivolumab, ipilimumab, atezolizumab, pembrolizumab, avelumab, or durvalumab use until April 11, 2018. Our search included combinations of the keyword "multiple sclerosis" with "nivolumab", "ipilimumab", "atezolizumab", "pembrolizumab", "durvalumab", and "avelumab". Two researchers performed this search independently for internal validity. Only three articles were found $[6,10,11]$, and were reviewed in full-text form. No language, time, age, or date filters were included in our search. 


\section{Statistics}

Categorical variables are presented in frequency and percentages. Continuous variables are presented with medians. Results were compared using Fisher's exact tests and Wilcoxon Rank Sum tests presented as p-values. The level of statistical significance was set at 0.05 for all tests conducted, and all analyses were performed with SPSS software version 23.

\section{RESULTS}

Our analysis of FAERS captured 42,529 adverse events with the use of ICI with 13 cases identified with MS. In the published literature our search identified three cases, but they were matched to cases in FAERS, as they contained identical clinical and treatment history, and were not counted as additional cases. We present an additional case from our practice further below.

The median age of presentation for MS cases identified was 52.5 years. ICI were used in the following indications (cancer type): melanoma $(n=7)$, non-small cell lung carcinoma in $(\mathrm{n}=2)$, pleural mesothelioma $(\mathrm{n}=1)$, renal cell carcinoma $(\mathrm{n}=1)$, and colo-rectal cancer $(\mathrm{n}=1)$. The indication was not reported in 2 patients.

The ICI with higher number of reported cases of MS was nivolumab ( $n=9)$, followed by ipilimumab $(n=5)$, pembrolizumab $(n=2)$, and atezolizumab $(n=1)$. No cases were attributed to durvalumab or avelumab. We found no differences while comparing agents or mechanism of action (PD-1 axis versus CTLA-4 inhibitors) between demographics or outcomes variables. History of MS was confirmed in 8 patients. One patient had a history of vitiligo, otherwise an autoimmune disorder history was negative or unknown. All of the patients had diagnosis of metastatic disease. Previous cancer therapy is largely unknown, but the cases that reported it received several courses of chemotherapy, surgery, and radiation prior to beginning immunotherapy. MS treatment was reported in 4 patients, 3 of which were previously receiving glatiramer acetate, and 1 patient was receiving interferon-beta. Of these patients, three were not receiving MS treatment while on ICI. The reason was to avoid possible interference with the mechanism of action (2 patients), withholding therapy before starting ICI, and because the diagnosis of MS was not clear before ICI therapy (1 patient).

Clinical presentation varied from paresthesias, vision changes, weakness, and altered mental status (table 2). A detailed description of the clinical presentation of case 10, 11, and 14 is available in the literature $[6,10,11]$, and case 5 was presented at the Society for Neuro Oncology Annual Meeting in 2016[12]. The basis for the diagnosis of MS was reported in 8 cases and was based on combination of clinical presentation, MRI brain studies ( 8 cases), biopsy ( 2 cases), and cerebrospinal fluid analyses ( 2 cases). Median time to symptom onset was 29 (12-120) days, with a median of 2 cycles to onset. Patients receiving CTLA-4 inhibitors had a numerically higher median time to onset of event, and number of cycles compared to PD-1/PD-L1 inhibitors, but was not statistically significant (Table 1). In all cases, the drug was discontinued when the clinical diagnosis of MS was clear. One patient was re-treated with ipilimumab after presenting mild symptoms of MS relapse, due to the beneficial cancer response. His MS symptoms significantly worsened following retreatment 
and his re-trial was discontinued. In two other cases, the course of MS relapse was particularly serious, with rapid progression and poor response to MS therapy.

Therapy for MS relapse was provided for 5 patients who received IV corticosteroids. Patients were not reported to have received any form of immunotherapy prior to or during immune checkpoint inhibitor administration. Symptom resolution was reported in 5 patients, with 3 patients presenting MS progression and significant disability, including 2 patients who died from their disease. Overall median duration of event as 56 (21-112) days. Median symptom duration was 4 weeks for PD axis inhibitors, with a trend to longer duration in CTLA-4 inhibitors that was not statistically significant (12 weeks compared to 4 weeks, $\mathrm{p}=0.700$ ) (Table 2). All cases presented with other serious adverse events and 4 patients required hospitalization.

\section{Case Report}

A 49-year-old woman with a history of relapsing remitting MS since 2003, and metastatic colonic adenocarcinoma since January 2015. She received off label use of atezolizumab with cobimetinib, a reversible inhibitor of mitogen-activated protein kinase/extracellular signal regulated kinase (MEK), in August 2016. Two weeks following single treatment she presented to the ED with a 3-day history of persistent fever and progressing confusion. She was admitted and underwent workup including brain and body imaging, laboratory tests, and cerebrospinal fluid (CSF) analysis. The laboratory workup was negative except for mild residual neutropenia and hyponatremia, and an increased total protein in CSF. An infectious cause was ruled out. Brain MRI on September 2016 demonstrated nonspecific T2 hyperintense lesions within the subcortical, deep, and periventricular white matter. No leptomeningeal enhancement was evident in the images. A presumptive diagnosis of MS relapse was made based on her history.

The patient's MS had been treated regularly with glatiramer acetate since 2009, with her last MS relapse in 2014. She was using glatiramer during her treatment with atezolizumab. Her cancer had been treated aggressively with multiple lines of chemotherapy, and surgical procedures (right colectomy, liver sectionectomy, and right hepatic artery radioembolization). Chemotherapy included FOLFOXIRI [5-fluorouracil (5-FU)/leucovorin, oxaliplatin, irinotecan] plus bevacizumab, 5-FU and bevacizumab, trifluridine and tipiracil, FOLFIRI (folinic acid, 5-FU, and irinotecan) plus bevacizumab, and regorafenib. The MS relapse being attributable to atezolizumab therapy was deemed probable based on the Naranjo Algorithm for Adverse Drug Reaction Probability Scale (Score=6)[29]. Her neurological status continued to deteriorate, showing no response to high-dose corticosteroids or supportive measures. Follow up imaging demonstrated a decreased size of liver metastases, but suspected disease progression versus pseudoprogression in the chest. Therapy was withheld due to her clinical status and she passed away a month after her first dose of atezolizumab.

\section{DISCUSSION}

Immune checkpoint inhibitors are thought to induce epitope spreading and a broader $\mathrm{T}$ cell response[22, 39], that may trigger immune-mediated syndromes. Previous analyses have 
demonstrated similar proliferation rates and pro-inflammatory cytokine production of myelin-reactive CD4+ T cells from ipilimumab treated patients, comparable to patients with MS[6]. In addition, in patients with a history of kidney transplantation that received ipilimumab, T-cell mediated graft rejection has been described[44].

The outcomes of patients presenting with neurological irAEs are thought to be favorable[7, 32], however there are few severe reactions described with severe organ failure leading to death $[7,8,46]$. Out of the fourteen cases, eight had a reported history of MS. The frequency of clinically isolated or radiographically isolated syndrome in patients that received immunotherapy is difficult to assess and would require a high level of suspicion in prospective studies. All of the patients in our analysis presented rapid disease progression requiring medical intervention, and two patients died from their MS relapse. Imaging, or pathological patterns, biopsy, or autopsy material was not available for our review, and is a limitation to FAERS data analysis.

The time to event for irAE remain an active area of investigation and may range from weeks to months[32]. Single center experiences and retrospective reviews describe a range of neurological irAEs that includes headaches, neuropathies, meningitis, and myasthenic syndromes $[7,18]$. This literature describes a median time to event of 6-13 weeks after therapy, with an incidence as high as $12 \%$ for reported neurological irAEs for combinatorial use of anti-PD1 and anti-CTLA-4 inhibitors[7, 14, 18].

The median of 29 days in our study, with few cases successfully responding to symptomatic treatment, suggests a rapid and aggressive reaction. Patients in our analysis had a median age of 52.5 years, younger than described for other neurological irAEs $(56-71$ years) [7, 18]. No clear association between ICI agent and neurological syndromes have been described to date[7], and in our analysis no demographic or outcome variables were found to be different between ICI agents. We believe the higher number of cases of nivolumab, and the higher number of cases with melanoma are related to earlier FDA approval, rather than agent or disease specific syndrome.

Specific cancer types may have a higher predisposition of developing neurological AEs. Neuropathies are more commonly associated with ANNA-1 antibodies, that are seen primarily in small cell lung cancer, breast cancer, and renal cancer[41]. The close temporal relationship of immune checkpoint inhibitors administration to the development of symptoms and documented MRI activity provide basis to support causation of immune checkpoint inhibitors to the MS relapse. Similar basis is used for other drugs related adverse events and is validated by the Naranjo Algorithm for Adverse Drug Reaction Probability Scale[29].

Eight patients had a history of MS in our analysis, of which 5 reported symptom resolution. A history of autoimmune disorders as a contraindication for ICI therapy is under debate, as some patients may still benefit from immune-based cancer therapies. Yet, it is expected that around $30 \%$ will either experience a flare of their disease or a new immunological event, generally with mild severity $[17,26]$. Limited evidence after solid organ transplantation suggests ICI should be used with caution, and may result in graft loss and life-threatening 
events[20, 21]. However, the reduction of immunosuppression has been questioned as the cause of graft loss[44]. Some patients had stopped MS therapy at the time they received immunotherapy. Nonetheless, the effect of immunomodulation of MS is not as predictable as immunosuppression after transplantation. Severe, life-threatening immune-based syndromes have been previously described after adjuvant exposure (vaccination, systemic viral infection) and ICI may trigger similar presentations[38].

Disease presentation was variable between cases, but was particularly severe in 3 cases, one of which was seen in our institution. Our patient presented a clinical syndrome resembling autoimmune encephalitis, with decreased level of consciousness and rapid progression. Immune activation cascade may differ from patient to patient[41], but much is still unknown on risk factors for developing neurological irAE. As the use of ICI increases, the number of patients presenting these reactions will increase.

Retrospective cohorts have demonstrated an adequate cancer response in patients that present irAEs[17]. Only two of our cohort were re-exposed or continued treatment. Interestingly, the majority of cases continued to manifest MS symptoms long after stopping therapy (Figure 1), likely related to the long half-life of ICI[7], and a prolonged and sustained immune response. A history of multiple rounds of cancer treatment, as was the case in the majority of these patients, may increase the risk and severity of neurotoxicities.

Some of the limitations of our study include the lack of detailed MS follow up, disability measures, and long outcome data. Reporting bias is a significant limitation of our sample, as cases with worst outcomes were more likely to be reported to the FDA. Larger analyses might confirm outcome differences between CTLA-4 inhibitors and PD-1 axis inhibitors in terms of MS relapse. Our analysis demonstrates ICI therapy in patients with MS may be associated with rapid worsening neurological condition and death. Future longitudinal studies to assess worsening neurologic function in patients with MS relapse after ICI therapy would provide information regarding prognosis and help outweigh the risks of ICI in these patients.

\section{Funding:}

Ms. Jayswal was supported by the Biostatistics and Bioinformatics Shared Resource of the University of Kentucky Markey Cancer Center (P30CA177558).

\section{REFERENCES}

1. Anonymous (2017) Global, regional, and national burden of neurological disorders during 19902015: a systematic analysis for the Global Burden of Disease Study 2015. The Lancet. Neurology 16:877-897 [PubMed: 28931491]

2. Ansell SM, Lesokhin AM, Borrello I et al. (2015) PD-1 blockade with nivolumab in relapsed or refractory Hodgkin's lymphoma. N Engl J Med 372:311-319 [PubMed: 25482239]

3. Ascherio A, Munger KL (2016) Epidemiology of Multiple Sclerosis: From Risk Factors to Prevention-An Update. Seminars in neurology 36:103-114 [PubMed: 27116717]

4. Borghaei H, Paz-Ares L, Horn L et al. (2015) Nivolumab versus Docetaxel in Advanced Nonsquamous Non-Small-Cell Lung Cancer. N Engl J Med 373:1627-1639 [PubMed: 26412456]

5. Brahmer J, Reckamp KL, Baas P et al. (2015) Nivolumab versus Docetaxel in Advanced SquamousCell Non-Small-Cell Lung Cancer. N Engl J Med 373:123-135 [PubMed: 26028407] 
6. Cao Y, Nylander A, Ramanan S et al. (2016) CNS demyelination and enhanced myelin-reactive responses after ipilimumab treatment. Neurology 86:1553-1556 [PubMed: 26984943]

7. Cuzzubbo S, Javeri F, Tissier M et al. (2017) Neurological adverse events associated with immune checkpoint inhibitors: Review of the literature. European journal of cancer (Oxford, England : 1990) 73:1-8

8. Fellner A, Makranz C, Lotem M et al. (2018) Neurologic complications of immune checkpoint inhibitors. J Neurooncol

9. Garon EB, Rizvi NA, Hui R et al. (2015) Pembrolizumab for the treatment of non-small-cell lung cancer. N Engl J Med 372:2018-2028 [PubMed: 25891174]

10. Gerdes LA, Held K, Beltran E et al. (2016) CTLA4 as Immunological Checkpoint in the Development of Multiple Sclerosis. Ann Neurol 80:294-300 [PubMed: 27351142]

11. Gettings EJ, Hackett CT, Scott TF (2015) Severe relapse in a multiple sclerosis patient associated with ipilimumab treatment of melanoma. Multiple sclerosis (Houndmills, Basingstoke, England) 21:670

12. Gómez Vicente L, Rubio Viqueira B, Jimenez De Las Peñas M et al. (2016) P04.07 Relapse in a paucisymptomatic form of multiple sclerosis in a patient treated with nivolumab. Neuro-oncology 18:iv25-iv25

13. Gulley JL, Madan RA, Pachynski R et al. (2017) Role of Antigen Spread and Distinctive Characteristics of Immunotherapy in Cancer Treatment. Journal of the National Cancer Institute 109

14. Haanen J, Carbonnel F, Robert C et al. (2017) Management of toxicities from immunotherapy: ESMO Clinical Practice Guidelines for diagnosis, treatment and follow-up. Annals of oncology : official journal of the European Society for Medical Oncology 28:iv119-iv142 [PubMed: 28881921]

15. Harinstein L, Wu E, Brinker A (2018) Postmarketing cases of eluxadoline-associated pancreatitis in patients with or without a gallbladder. Alimentary pharmacology \& therapeutics 47:809-815 [PubMed: 29349789]

16. Herbst RS, Baas P, Kim DW et al. (2016) Pembrolizumab versus docetaxel for previously treated, PD-L1-positive, advanced non-small-cell lung cancer (KEYNOTE-010): a randomised controlled trial. Lancet 387:1540-1550 [PubMed: 26712084]

17. Johnson DB, Sullivan RJ, Ott PA et al. (2016) Ipilimumab Therapy in Patients With Advanced Melanoma and Preexisting Autoimmune Disorders. JAMA oncology 2:234-240 [PubMed: 26633184]

18. Kao JC, Liao B, Markovic SN et al. (2017) Neurological Complications Associated With AntiProgrammed Death 1 (PD-1) Antibodies. JAMA neurology 74:1216-1222 [PubMed: 28873125]

19. Khoury SJ, Sayegh MH (2004) The roles of the new negative T cell costimulatory pathways in regulating autoimmunity. Immunity 20:529-538 [PubMed: 15142522]

20. Kuo JC, Lilly LB, Hogg D (2018) Immune checkpoint inhibitor therapy in a liver transplant recipient with a rare subtype of melanoma: a case report and literature review. Melanoma research 28:61-64 [PubMed: 29140833]

21. Kwatra V, Karanth NV, Priyadarshana K et al. (2017) Pembrolizumab for metastatic melanoma in a renal allograft recipient with subsequent graft rejection and treatment response failure: a case report. Journal of medical case reports 11:73 [PubMed: 28315636]

22. Kwek SS, Dao V, Roy R et al. (2012) Diversity of antigen-specific responses induced in vivo with CTLA-4 blockade in prostate cancer patients. Journal of immunology (Baltimore, Md. : 1950) 189:3759-3766

23. Larkin J, Chmielowski B, Lao CD et al. (2017) Neurologic Serious Adverse Events Associated with Nivolumab Plus Ipilimumab or Nivolumab Alone in Advanced Melanoma, Including a Case Series of Encephalitis. The oncologist 22:709-718 [PubMed: 28495807]

24. Larkin J, Hodi FS, Wolchok JD (2015) Combined Nivolumab and Ipilimumab or Monotherapy in Untreated Melanoma. The New England journal of medicine 373:1270-1271

25. Liao B, Shroff S, Kamiya-Matsuoka C et al. (2014) Atypical neurological complications of ipilimumab therapy in patients with metastatic melanoma. Neurooncology 16:589-593 
26. Menzies AM, Johnson DB, Ramanujam S et al. (2017) Anti-PD-1 therapy in patients with advanced melanoma and preexisting autoimmune disorders or major toxicity with ipilimumab. Ann Oncol 28:368-376 [PubMed: 27687304]

27. Metz I, Weigand SD, Popescu BF et al. (2014) Pathologic heterogeneity persists in early active multiple sclerosis lesions. Annals of neurology 75:728-738 [PubMed: 24771535]

28. Motzer RJ, Escudier B, Mcdermott DF et al. (2015) Nivolumab versus Everolimus in Advanced Renal-Cell Carcinoma. N Engl J Med 373:1803-1813 [PubMed: 26406148]

29. Naranjo CA, Busto U, Sellers EM et al. (1981) A method for estimating the probability of adverse drug reactions. Clinical pharmacology and therapeutics 30:239-245 [PubMed: 7249508]

30. Noseworthy JH, Lucchinetti C, Rodriguez M et al. (2000) Multiple sclerosis. The New England journal of medicine 343:938-952 [PubMed: 11006371]

31. Numico G, Cristofano A, Mozzicafreddo A et al. (2015) Hospital admission of cancer patients: avoidable practice or necessary care? PloS one 10:e0120827 [PubMed: 25812117]

32. Postow MA, Sidlow R, Hellmann MD (2018) Immune-Related Adverse Events Associated with Immune Checkpoint Blockade. The New England journal of medicine 378:158-168 [PubMed: 29320654]

33. Ramagopalan SV, Sadovnick AD (2011) Epidemiology of multiple sclerosis. Neurologic clinics 29:207-217 [PubMed: 21439437]

34. Robert C, Long GV, Brady B et al. (2015) Nivolumab in previously untreated melanoma without BRAF mutation. The New England journal of medicine 372:320-330 [PubMed: 25399552]

35. Robert C, Schachter J, Long GV et al. (2015) Pembrolizumab versus Ipilimumab in Advanced Melanoma. The New England journal of medicine 372:2521-2532 [PubMed: 25891173]

36. Sakaeda T, Tamon A, Kadoyama K et al. (2013) Data mining of the public version of the FDA Adverse Event Reporting System. International journal of medical sciences 10:796-803 [PubMed: 23794943]

37. Sarangdhar M, Tabar S, Schmidt C et al. (2016) Data mining differential clinical outcomes associated with drug regimens using adverse event reporting data. Nature biotechnology 34:697700

38. Shoenfeld Y, Agmon-Levin N (2011) 'ASIA' - autoimmune/inflammatory syndrome induced by adjuvants. Journal of autoimmunity 36:4-8 [PubMed: 20708902]

39. Snyder A, Makarov V, Merghoub T et al. (2014) Genetic basis for clinical response to CTLA-4 blockade in melanoma. The New England journal of medicine 371:2189-2199 [PubMed: 25409260]

40. Spain L, Walls G, Julve M et al. (2017) Neurotoxicity from immune-checkpoint inhibition in the treatment of melanoma: a single centre experience and review of the literature. Annals of oncology : official journal of the European Society for Medical Oncology 28:377-385 [PubMed: 28426103]

41. Tobin WO, Pittock SJ (2017) Autoimmune Neurology of the Central Nervous System. Continuum (Minneapolis, Minn.) 23:627-653

42. Voskens CJ, Goldinger SM, Loquai C et al. (2013) The price of tumor control: an analysis of rare side effects of anti-CTLA-4 therapy in metastatic melanoma from the ipilimumab network. PloS one 8:e53745 [PubMed: 23341990]

43. Weiner HL (2004) Multiple sclerosis is an inflammatory T-cell-mediated autoimmune disease. Archives of neurology 61:1613-1615 [PubMed: 15477521]

44. Zehou O, Leibler C, Arnault JP et al. (2018) Ipilimumab for the treatment of advanced melanoma in six kidney transplant patients. American journal of transplantation : official journal of the American Society of Transplantation and the American Society of Transplant Surgeons

45. Zimmer L, Goldinger SM, Hofmann L et al. (2016) Neurological, respiratory, musculoskeletal, cardiac and ocular side-effects of anti-PD-1 therapy. European journal of cancer (Oxford, England : 1990) 60:210-225

46. Zukas AM, Schiff D (2018) Neurological complications of new chemotherapy agents. Neurooncology 20:24-36 [PubMed: 28992326] 


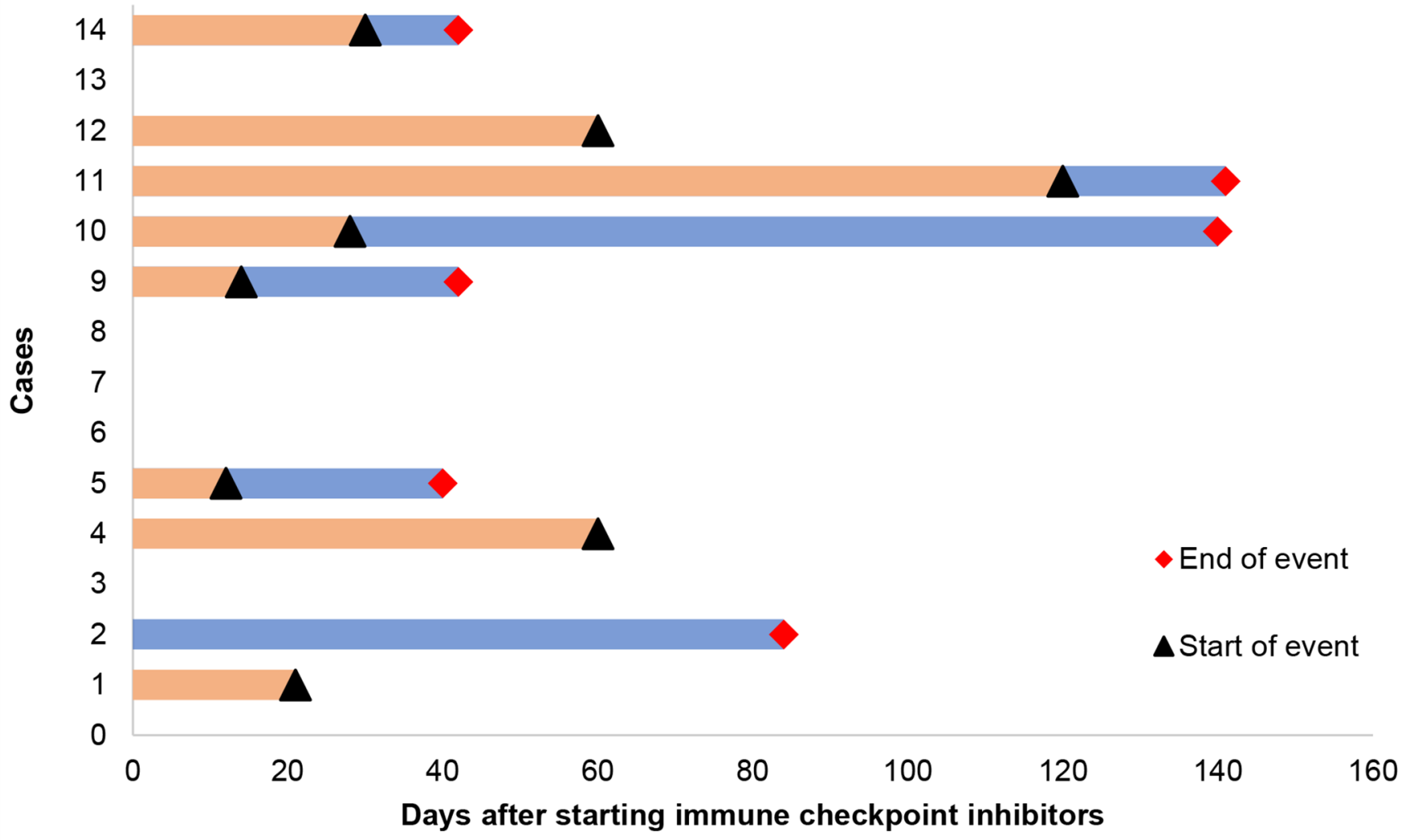

Figure 1.

Swimmers plot for duration of ICI therapy and MS symptoms. The orange line depicts the time since starting an ICI to the time that MS symptoms began. The blue line depicts the duration of symptoms. 
Table 1.

Overall demographics and characteristics by patients (TTE: Time to event)

\begin{tabular}{|c|c|c|l|l|l|l|l|l|l|l|l|}
\hline Case & Age & Gender & Indication & ICI & $\begin{array}{l}\text { Hx } \\
\text { of } \\
\text { MS }\end{array}$ & TTE & CTE & $\begin{array}{l}\text { Total } \\
\text { Cycles }\end{array}$ & Outcome & Resolution & Duration \\
\hline $\mathbf{1}$ & 58 & F & Melanoma & Pembrolizumab & Yes & 3 wks & 1 & 3 & OT & Yes & NR \\
\hline $\mathbf{2}$ & NR & M & $\begin{array}{l}\text { Pleural } \\
\text { mesothelioma }\end{array}$ & Pembrolizumab & NR & NR & NR & NR & HO, OT & No & 3 mo \\
\hline $\mathbf{3}$ & 50 & F & Melanoma & Nivolumab & Yes & NR & NR & NR & OT & NR & NR \\
\hline $\mathbf{4}$ & 63 & F & NSCLC & Nivolumab & NR & 2 mo & 2 & 2 & OT & NR & NR \\
\hline $\mathbf{5}$ & 43 & F & NSCLC & Nivolumab & Yes & 12 d & 1 & 1 & HO, OT & Yes & $1 \mathrm{mo}$ \\
\hline 6 & NR & NR & NR & Nivolumab & NR & NR & NR & NR & OT & NR & NR \\
\hline $\mathbf{7}$ & NR & NR & NR & Nivolumab & NR & NR & 2 & 2 & OT & NR & NR \\
\hline $\mathbf{8}$ & 45 & M & RCC & Nivolumab & No & NR & NR & NR & OT & NR & NR \\
\hline $\mathbf{9}$ & 49 & F & Colorectal & Atezolizumab & Yes & 2 wks & 1 & 1 & DE, HO & No & $1 \mathrm{mo}$ \\
\hline $\mathbf{1 0}$ & 76 & F & Melanoma & Ipilimumab & No & 4 wks & 4 & 4 & DE & No & $4 \mathrm{mo}$ \\
\hline $\mathbf{1 1}$ & 29 & M & Melanoma & Ipilimumab & Yes & 4 mo & 8 & 8 & OT, HO & Yes & 3 wks \\
\hline $\mathbf{1 2}$ & 55 & M & Melanoma & Ipilimumab & Yes & 2 mo & 3 & 3 & OT & Yes & NR \\
\hline $\mathbf{1 3}$ & NR & M & Melanoma & Ipilimumab & Yes & NR & NR & NR & OT & NR & NR \\
\hline $\mathbf{1 4}$ & 56 & M & Melanoma & Ipilimumab & Yes & 1 mo & 4 & 4 & OT & Yes & $3 \mathrm{mo}$ \\
\hline
\end{tabular}

ICI: Immune checkpoint inhibitor, Hx: History; MS: Multiple sclerosis; TTE: Time to event, CTE: Number of cycles to event, wks: Weeks; mo: Months; d: days 
Table 2.

Clinical phenotype descriptions as available in the reports

\begin{tabular}{|c|l|}
\hline Patient & Clinical presentation (as described in the reports) \\
\hline $\mathbf{1}$ & Paresthesia and decreased strength in lower and upper limbs. \\
\hline $\mathbf{3}$ & Numbness to hand a feet and loss of bladder control. \\
\hline $\mathbf{5}$ & Progressive diplopia, gait instability, bilateral ptosis, internuclear opthalmoplegia, and ataxic gait. \\
\hline $\mathbf{9}$ & Fever and progressive confusion. \\
\hline $\mathbf{1 0}$ & Fatigue, memory loss, and vision changes. \\
\hline $\mathbf{1 1}$ & Thermhypaesthesia of both feet. \\
\hline $\mathbf{1 2}$ & Left leg weakness, left arm weakness, and significant fatigue \\
\hline $\mathbf{1 4}$ & Left lower extremity hemiparesis, gait dysfunction, and ataxia. \\
\hline
\end{tabular}


Table 3.

Demographics and characteristics by mechanism of action of immune checkpoint inhibitor

\begin{tabular}{|c|c|c|c|c|c|c|}
\hline & & \multicolumn{2}{|c|}{ CTLA-4 N=5 } & \multicolumn{2}{|c|}{ PD-1/PD-L1 N= 9} & p-value \\
\hline Age (years) & Median & \multicolumn{2}{|r|}{56} & \multicolumn{2}{|c|}{46} & 0.762 \\
\hline TTE (days) & Median & \multicolumn{2}{|r|}{30} & \multicolumn{2}{|c|}{13} & 0.114 \\
\hline Cycles TE & Median & \multicolumn{2}{|r|}{4} & \multicolumn{2}{|c|}{1} & 0.111 \\
\hline Total cycles & Median & \multicolumn{2}{|r|}{4} & \multicolumn{2}{|c|}{1} & 0.016 \\
\hline Duration of event (weeks) & Median & \multicolumn{2}{|r|}{12} & \multicolumn{2}{|c|}{4} & 0.700 \\
\hline & & $\mathbf{N}$ & $\%$ & $\mathbf{N}$ & $\%$ & \\
\hline \multirow[t]{3}{*}{ Gender } & Female & 1 & $20.0 \%$ & 5 & $55.6 \%$ & 0.236 \\
\hline & Male & 4 & $80.0 \%$ & 2 & $22.2 \%$ & \\
\hline & Not reported & 0 & $0.0 \%$ & 2 & $22.2 \%$ & \\
\hline \multirow[t]{3}{*}{ History of MS } & No & 1 & $20.0 \%$ & 1 & $11.1 \%$ & 0.301 \\
\hline & Yes & 4 & $80.0 \%$ & 4 & $44.4 \%$ & \\
\hline & Not reported & 0 & $0.0 \%$ & 4 & $44.4 \%$ & \\
\hline \multirow[t]{2}{*}{ OT } & No & 1 & $20.0 \%$ & 1 & $11.1 \%$ & 0.604 \\
\hline & Yes & 4 & $80.0 \%$ & 8 & $88.9 \%$ & \\
\hline \multirow[t]{2}{*}{ DE } & No & 4 & $80.0 \%$ & 8 & $88.9 \%$ & 0.604 \\
\hline & Yes & 1 & $20.0 \%$ & 1 & $11.1 \%$ & \\
\hline \multirow[t]{2}{*}{ HO } & No & 4 & $80.0 \%$ & 6 & $66.7 \%$ & 0.545 \\
\hline & Yes & 1 & $20.0 \%$ & 3 & $33.3 \%$ & \\
\hline \multirow[t]{3}{*}{ Resolution } & No & 1 & $20.0 \%$ & 2 & $22.2 \%$ & 0.513 \\
\hline & Yes & 3 & $60.0 \%$ & 2 & $22.2 \%$ & \\
\hline & Not reported & 1 & $20.0 \%$ & 5 & $55.6 \%$ & \\
\hline \multirow[t]{2}{*}{ Corticosteroids } & Not reported & 3 & $60.0 \%$ & 6 & $66.7 \%$ & 0.622 \\
\hline & Yes & 2 & $40.0 \%$ & 3 & $33.3 \%$ & \\
\hline
\end{tabular}

p-values are from Fisher's exact tests and Wilcoxon Rank-Sum.

TTE: Time to event; TE: To event; MS: Multiple sclerosis; OT: Other serious complication; DE: Death; HO: Hospitalization 